\title{
Laser-shock compression of magnesium oxide in the warm-dense-matter regime
}

\author{
K. Miyanishi, ${ }^{1,{ }^{*}}$ Y. Tange,,${ }^{2,3,4, \dagger}$ N. Ozaki,${ }^{1,5, \ddagger}$ T. Kimura, ${ }^{3}$ T. Sano, ${ }^{6}$ Y. Sakawa,${ }^{6}$ T. Tsuchiya,${ }^{3,4}$ and R. Kodama ${ }^{1,5}$ \\ ${ }^{1}$ Graduate School of Engineering, Osaka University, Suita, Osaka 565-0871, Japan \\ ${ }^{2}$ Japan Synchrotron Radiation Research Institute, Sayo, Hyogo 679-5198, Japan \\ ${ }^{3}$ Geodynamics Research Center, Ehime University, Matsuyama, Ehime 790-8577, Japan \\ ${ }^{4}$ Earth-Life Science Institute Ehime Satellite, Tokyo Institute of Technology, Meguro, Tokyo 152-8550, Japan \\ ${ }^{5}$ Photon Pioneers Center, Osaka University, Suita, Osaka 565-0871, Japan \\ ${ }^{6}$ Institute of Laser Engineering, Osaka University, Suita, Osaka 565-0871, Japan
}

(Received 3 October 2014; revised manuscript received 27 February 2015; published 17 August 2015)

\begin{abstract}
Magnesium oxide has been experimentally and computationally investigated in the warm-dense solid and liquid ranges from $200 \mathrm{GPa}$ to $1 \mathrm{TPa}$ along the principal Hugoniot. The linear approximation between shock velocity and particle velocity is validated up to a shock velocity of $15 \mathrm{~km} / \mathrm{s}$ from the experimental data, this suggesting that the $\mathrm{MgO} \mathrm{B} 1$ structure is stable up to the corresponding shock pressure of $\sim 350 \mathrm{GPa}$. Moreover, our Hugoniot data, combined with ab initio simulations, show two crossovers between MgO Hugoniot and the extrapolation of the linear approximation line, occurring at a shock pressures of approximately 350 and $650 \mathrm{GPa}$, with shock temperatures of 8000 and $14000 \mathrm{~K}$, respectively. These crossover regions are consistent with the solid-solid (B1-B2) and the solid-liquid (B2-melt) phase boundaries predicted by the ab initio calculations.
\end{abstract}

DOI: 10.1103/PhysRevE.92.023103

PACS number(s): 52.35.Tc, 64.30.Jk, 52.30.-q, 52.90.+z

\section{INTRODUCTION}

Various types of exoplanets, such as super-Earths, which do not exist in our solar system, have been discovered thanks to advances in planetary observational technology. These types of objects are considered to be important for developing a more general theory of planet formation. Magnesium oxide $(\mathrm{MgO})$ or periclase is believed to be one of the most common materials constituting the interior of such exoplanets.

$\mathrm{MgO}$ has a simple $\mathrm{NaCl}$ (B1-type) structure up to at least $200 \mathrm{GPa}[1,2]$, and it also has a very high melting temperature above $3000 \mathrm{~K}[3,4]$. Because of the broad stability range, the pressure-volume-temperature equation of state (EOS) of $\mathrm{MgO}$ has been used as an internal pressure standard for high-pressure and high-temperature static experiments $[5,6]$. In general, B1-type materials are known to transform into B2-type ( $\mathrm{CsCl}$ structure) [7-13]. The B1-B2 phase transitions have been investigated intensively both in statically highpressure experiments and theoretical calculations. However, in the case of $\mathrm{MgO}$, the phase transition had not been observed experimentally until recently due to the technical difficulties, while theoretical simulations had predicted the transition occurring at multi-Mbar (multi-100 GPa) pressure conditions [14-16].

Recent technical developments in dynamic compression experiments using high-power lasers have allowed us to access extremely high-pressure conditions even for less dense materials. The phase transitions of $\mathrm{MgO}$ along shock compression and ramp compression curves were recently implied [17,18]. McWilliams et al. observed two temperature anomalies on smoothly decaying shocked $\mathrm{MgO}$ at $\sim 14000 \mathrm{~K}(\sim 600 \mathrm{GPa})$ and $\sim 8000 \mathrm{~K}(\sim 400 \mathrm{GPa})$, interpreting those as the melting and the solid-solid phase transitions (e.g., B1-B2) along

\footnotetext{
*miyanishi@ef.eie.eng.osaka-u.ac.jp

†yoshinori.tange@spring8.or.jp

${ }^{\ddagger}$ norimasa.ozaki@eei.eng.osaka-u.ac.jp
}

the shock Hugoniot, respectively [17]. These experiments triggered controversies over the phase boundaries at the multiMbar pressures in the latest $a b$ initio calculations [19,20]. In the laser-shock compression study, the $\mathrm{MgO}$ pressures were determined by extrapolating the $\mathrm{MgO}$ principal Hugoniot up to above $1 \mathrm{TPa}$ using the available low-pressure data from below $200 \mathrm{GPa}[1,21,22]$. The lack of experimental data over such a wide range of pressure and temperature is a significant problem. New EOS data of $\mathrm{MgO}$ in the broad warm-dense-matter regime are needed to compare with the theoretical simulation results and to understand the phase relations.

Additionally, it is necessary to reveal the phase relations of $\mathrm{MgO}$ as not only planetary material but also pressure standard material. The EOS data for $\mathrm{MgO}$ in the warm-dense-matter regime [as opposed to the regions of cold solid or hot plasma (atomic fluid)], play a critical role for better understanding the behavior of the pressure standard material at high pressures and high temperatures in static experiments.

In this paper, we present measurements of the principal Hugoniot EOS and shock temperature for MgO from $200 \mathrm{GPa}$ to $1 \mathrm{TPa}$. Our laser-shock data, combined with $a b$ initio simulations, help to understand the states of the warm-dense $\mathrm{MgO}$ and the phase relations.

\section{PROCEDURES}

\section{A. Laser-shock experiments}

The laser-driven shock experiments on $\mathrm{MgO}$ were performed with the GEKKO XII laser facility at the Osaka University. The facility is a neodymium-doped glass laser system. Three laser beams were frequency-doubled to a wavelength of $527 \mathrm{~nm}$, and nine laser beams were frequencytripled to a wavelength of $351 \mathrm{~nm}$. Laser energies up to $\sim 500 \mathrm{~J}$ were delivered onto a focal spot to generate the shock pressures into a target. The temporal shape of the laser pulse was approximately square with a $2.5 \mathrm{~ns}$ duration [full width at half-maximum (FWHM)] and rise and fall times of $100 \mathrm{ps}$ 


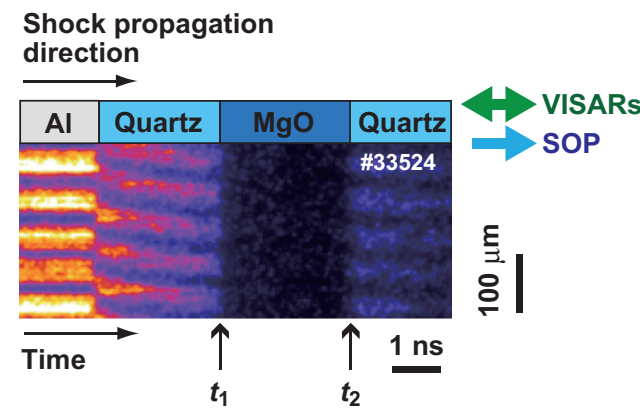

FIG. 1. (Color online) A typical raw VISAR trace, with schematics of a target assembly and experimental geometry.

each. The focal-spot diameter was typically $600 \mu \mathrm{m}$ or $1 \mathrm{~mm}$ with a flat-top distribution resulting in a planar shock front more than $400 \mu \mathrm{m}$ in diameter [23].

A single crystal of $\mathrm{MgO}$ with a thickness of $20-50 \mu \mathrm{m}$ was used as a sample. The $\mathrm{MgO}$ crystal was polished on both sides along the (100) plane, and it had a fine surface quality of $\lambda / 4$ for a frequency-doubled YAG laser $(\lambda=532 \mathrm{~nm})$. The initial density $\rho_{0}$ and temperature $T_{0}$ of $\mathrm{MgO}$ are $3.58 \mathrm{~g} / \mathrm{cm}^{3}$ and $\sim 300 \mathrm{~K}$, respectively. A typical target assembly consisted of polypropylene $(\mathrm{CH})$, aluminum $(\mathrm{Al}), \alpha$-quartz $\left(\mathrm{SiO}_{2}\right)$, and $\mathrm{MgO}$ from the laser irradiation side. A schematic of the target package and the experimental arrangement are shown in Fig. 1. The $\mathrm{CH}$ polymer was used as an ablator to generate a shock wave and to minimize hard-x-ray emissions in the laser-plasma interaction region. The quartz was a reference material of the principal Hugoniot for the impedance matching analysis [24].

The impedance matching analysis is, ideally, performed using the quartz shock velocity and the $\mathrm{MgO}$ shock velocity just before and after shock arrival to the quartz-MgO interface. However, it was difficult to measure the instantaneous shock velocity of $\mathrm{MgO}$, because $\mathrm{MgO}$ did not become highly reflecting in the warm-dense-matter regime. $\mathrm{MgO}$ was only partially transparent at pressures even up to $\sim 400 \mathrm{GPa}$, and it gradually became opaque with increasing shock pressure. Therefore, the average shock velocity of $\mathrm{MgO}$ was determined in this experiment from the measurements of $\mathrm{MgO}$ sample thickness and shock transit time. If significant shock decay occurs in the $\mathrm{MgO}$ layer, our average shock velocity can be very different from the instantaneous shock velocity. For example, a $10 \%$ decay of shock velocity generates a $5 \%$ difference between the average and instantaneous velocity. This influences the locus of the Hugoniot point of $\mathrm{MgO}$ significantly, therefore we used thin enough $\mathrm{MgO}$ samples here to minimize this uncertainty. Moreover, we used another $\alpha$-quartz, glued onto the rear side of the $\mathrm{MgO}$ sample, as a sensor for exactly determining the shock arrival time to the $\mathrm{MgO}$ rear surface and precisely evaluating the decaying effect because quartz became a good optical reflector under shock compression above $\sim 150 \mathrm{GPa}$. A typical VISAR image is shown in Fig. 1. At times $t_{1}$ and $t_{2}$ in the figure, the shock wave arrives at the quartz- $\mathrm{MgO}$ and $\mathrm{MgO}$-quartz interfaces, respectively. The shock velocity in the front side quartz, $U_{s}^{q 1}$, was stabilized gradually with time, and the shock decaying factor from the derivative of the shock velocity, $d U_{s}^{q 1} / d t$, was $\sim-0.8 \mathrm{~km} / \mathrm{ns}^{2}$ just before $t=t_{1}$. The shock in the rear quartz,
$U_{s}^{q 2}$, was fairly steady, and the factor was $\sim-0.3 \mathrm{~km} / \mathrm{ns}^{2}$ just after $t=t_{2}$. The use of the target assembly and analysis allows us to obtain relatively accurate shock velocity data even in the optically opaque region of $\mathrm{MgO}$. Since the temporal resolution was $\sim 70$ ps in this measurement, the uncertainty of the shock transit time was typically $2-3 \%$.

$\mathrm{MgO}$ Hugoniots were determined using the $\alpha$-quartz reference $[24,25]$ on the basis of the impedance matching analysis [26]. Shock velocities $\left(U_{s}\right)$ of $\mathrm{MgO}$ and quartz were measured with two line-imaging velocity interferometers (line-VISARs) [27], and the particle velocity $\left(u_{p}\right)$ of $\mathrm{MgO}$ was determined by matching shock impedances of the $\mathrm{MgO}$ and quartz. Shock pressure $P$, density $\rho$, and internal energy $E$ in $\mathrm{MgO}$ were determined from the $U_{s}$ and $u_{p}$ using basic conservation relations (Rankine-Hugoniot relations) [26].

The shock temperatures were measured with a streaked optical pyrometer (SOP) [28]. The SOP was calibrated based on the known shock temperatures in quartz [29,30], and it was spatially and temporally resolved to image the selfemission from the shocked matter around a $455 \mathrm{~nm}$ wavelength with a narrow bandwidth (38 nm FWHM), providing the brightness temperature. In the less reflecting region of $\mathrm{MgO}$, we determined the shock temperatures assuming that the shocked $\mathrm{MgO}$ behaved as a blackbody radiator.

\section{B. $A b$ initio calculations}

Our $a b$ initio calculations are based on the densityfunctional theory and the local-density approximation [31], where wave functions are expanded on a plane-wave basis and electronic structures are solved using pseudopotentials, the details of which are given in our previous study [32]. The solid-solid phase boundary between the B1 and B2 phases is calculated based on the lattice dynamics method combined with the quasiharmonic approximation. These techniques are basically similar to those in the previous studies on the ultrahigh-pressure phase relation of $\mathrm{MgO}[14,15,19,20]$, however here the lattice dynamics calculations for determining the solid-solid phase boundary were performed using the density-functional perturbation theory [32]. This can calculate phonon free energies with a higher numerical precision compared to the direct lattice dynamics method $[15,20]$ and Fourier analyses of velocity autocorrelation functions obtained from molecular dynamics [19].

The two-phase coexisting method was applied to determine the melting temperatures of both B1 and B2 phases in the same way as in our previous study on the melting phase relation of $\mathrm{SiO}_{2}$ [33]. Computation conditions for the $\mathrm{B} 1$ phase are almost the same as in Refs. [14] and [15], so that the results agree well with each other, while the two-phase coexisting method was applied to the B2 phase with a $4 \times 4 \times 8$ supercell (256 atoms).

\section{RESULTS AND DISCUSSION}

\section{A. Physical properties along the principal Hugoniot}

A total of seven shots were conducted, and shock Hugoniot data at $\sim 180-950 \mathrm{GPa}$ were obtained (Table I). The $U_{s}-u_{p}$ relationship of $\mathrm{MgO}$ was determined at the pressures along the principal Hugoniot, as shown in Fig. 2. We found that our experimental data below a shock velocity of $\sim 15 \mathrm{~km} / \mathrm{s}$, 
TABLE I. Shock compression data for MgO. $\eta$ denotes density compressibility, where $\rho_{0}$ is the initial density.

\begin{tabular}{|c|c|c|c|c|c|c|}
\hline $\begin{array}{l}\text { Shot } \\
\text { No. }\end{array}$ & $\begin{array}{c}U_{s} \\
(\mathrm{~km} / \mathrm{s})\end{array}$ & $\begin{array}{c}u_{p} \\
(\mathrm{~km} / \mathrm{s})\end{array}$ & $\begin{array}{c}P \\
(\mathrm{GPa})\end{array}$ & $\begin{array}{c}\rho(=1 / V) \\
\left(\mathrm{g} / \mathrm{cm}^{3}\right)\end{array}$ & $\eta\left(=\rho / \rho_{0}\right)$ & $\begin{array}{c}T \\
(\mathrm{~K})\end{array}$ \\
\hline 33713 & $12.45 \pm 0.53$ & $4.11 \pm 0.16$ & $183.5 \pm 10.6$ & $5.35 \pm 0.15$ & $1.49 \pm 0.04$ & - \\
\hline 33544 & $13.85 \pm 0.40$ & $5.61 \pm 0.16$ & $278.4 \pm 11.3$ & $6.02 \pm 0.17$ & $1.68 \pm 0.05$ & - \\
\hline 33698 & $14.75 \pm 0.32$ & $6.18 \pm 0.25$ & $326.7 \pm 18.0$ & $6.18 \pm 0.31$ & $1.72 \pm 0.07$ & - \\
\hline 33720 & $15.43 \pm 0.83$ & $7.22 \pm 0.37$ & $399.5 \pm 29.7$ & $6.74 \pm 0.44$ & $1.88 \pm 0.12$ & - \\
\hline 33524 & $18.22 \pm 0.72$ & $9.36 \pm 0.43$ & $610.9 \pm 37.2$ & $7.37 \pm 0.47$ & $2.06 \pm 0.13$ & $13600 \pm 1730$ \\
\hline 33684 & $19.98 \pm 0.59$ & $9.82 \pm 0.44$ & $702.9 \pm 37.5$ & $7.04 \pm 0.36$ & $1.97 \pm 0.10$ & $16120 \pm 2470$ \\
\hline 33509 & $22.29 \pm 0.73$ & $11.90 \pm 0.48$ & $950.7 \pm 49.6$ & $7.69 \pm 0.46$ & $2.15 \pm 0.13$ & $23120 \pm 3760$ \\
\hline
\end{tabular}

(a)

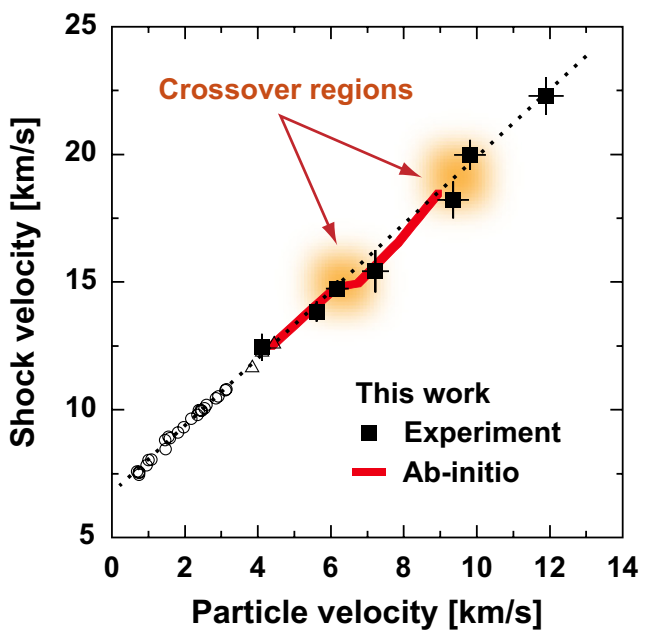

(b)

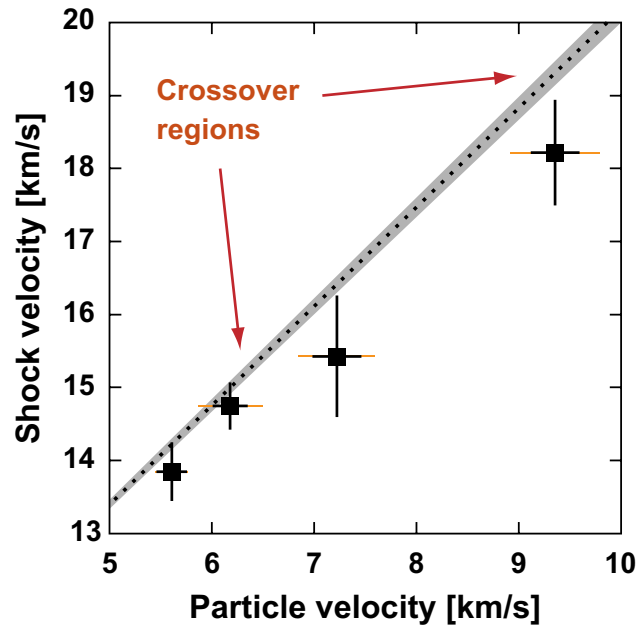

FIG. 2. (Color online) Shock velocity-particle velocity relationships of $\mathrm{MgO}$ up to $1 \mathrm{TPa}$. (a) Present experimental results (solid squares) are shown with ab initio calculation results (thick solid line), previous results reported in LASL [21] (open circles), those by Vassiliou and Ahrens [1] (open triangles), and the linear-fit extrapolation (dotted line) employed by McWilliams et al. [17]. (b) Magnified view of (a) near the first crossover region. The hatched dotted line represents the present linear relation and its uncertainty. Each data point from this study is given by a pair of overlapping error bars with the larger errors (lighter colored) representing the total uncertainty and the smaller errors representing the random uncertainty. The total uncertainty corresponds to a quadrature sum of both random (arising from velocity measurements) and systematic (arising from the uncertainty of quartz EOS) uncertainties. corresponding to a shock pressure of $\sim 320 \mathrm{GPa}$, are in excellent agreement with the extrapolation line of the $U_{s}-u_{p}$ linear fit to the previous low-pressure data (dotted line in the figure) $[1,21]$. The $U_{s}-u_{p}$ relationship is $U_{s}=(6.621 \pm$ $0.049)+(1.356 \mp 0.023) u_{p} \mathrm{~km} / \mathrm{s}$, calculated including our three low-pressure data, as shown in Fig. 2(b). In contrast, significant disagreements between the experimental data and the linear-fit extrapolation were found above this shock velocity, with the deviation of the data from the linear relation being more than 5\%. The low-pressure Hugoniot data verify the linear extrapolation, suggesting that the initial structure of $\mathrm{MgO}$ (B1 phase) is stable up to the shock velocity of $\sim 15 \mathrm{~km} / \mathrm{s}$ and the corresponding shock pressure of $\sim 320 \mathrm{GPa}$, and the higher pressure data suggest that two crossovers between the $\mathrm{MgO}$ Hugoniot and the linear extrapolation exist at shock velocities of $\sim 15$ and $\sim 18 \mathrm{~km} / \mathrm{s}$, highlighted by arrows in Fig. 2(a).

Figure 3 shows the present shock Hugoniot data and $a b$ initio calculations in pressure-density compression space. The $a b$ initio calculation results (thick solid line in the figure)

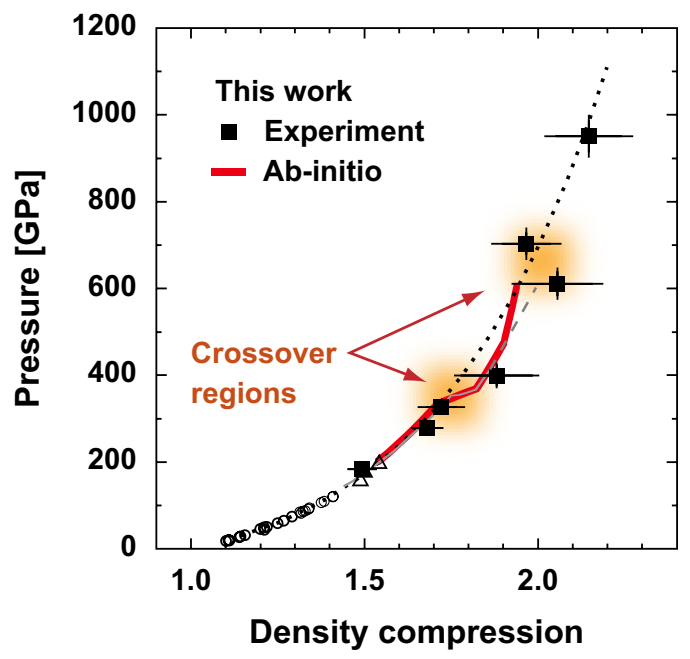

FIG. 3. (Color online) Shock pressure-density compressibility relationship of $\mathrm{MgO}$ up to $1 \mathrm{TPa}$. Present experimental results (solid squares) are shown with $a b$ initio calculation results (thick line), the previous experimental results reported in LASL [21] (open circles), those by Vassiliou and Ahrens [1] (open triangles), the previous $a b$ initio results by Cebulla and Redmer [20] (dashed line), and the $U_{s}-u_{p}$ linear-fit extrapolation (dotted line) employed by McWilliams et al. [17]. 
indicate a $7 \%$ density jump at $\sim 350 \mathrm{GPa}$ (i.e., the first crossover region) and a stiff behavior of the $\mathrm{MgO}$ Hugoniot after the density jump (in the region between two crossovers), which is consistent with the present experimental Hugoniot data. In the pressure range between 400 and $700 \mathrm{GPa}$, the mean density was $7.05 \mathrm{~g} / \mathrm{cm}^{3}$ from the experimental data. Using this mean density, we estimated the average lattice size of $\mathrm{MgO}$ in this pressure range from the equation $\sqrt[3]{Z / \bar{\rho} A}$, where $Z$ is the atomic weight of the $\mathrm{MgO}$ lattice unit cell, $\bar{\rho}$ is the mean density in $\mathrm{g} / \mathrm{cm}^{3}$, and $A$ is the Avogadro number. The average lattice size is $2.24 \AA$ assuming a B2 structure in this range. This value is consistent with the $d$-spacing of $\mathrm{B} 2$ around the phase transformation point ( $\sim 600 \mathrm{GPa})$ along the ramp compression proposed by Coppari et al. [18].

This might imply that the solid-solid phase transformation of $\mathrm{MgO}$ occurs under short duration pressure loading, such as a laser-driven shock wave. The duration of the shock in this case can be considered to be a few nanoseconds, comparable to the pulse duration of the shock-drive laser. It is probably possible for $\mathrm{MgO}$ to complete the phase transformation on such an ultrashort time scale if the displacive transformations predicted by numerical simulations [35] could be induced. The present work strongly motivates an additional experiment in which ultrafast XRD observations are performed using a femtosecond $\mathrm{X}$-ray pulse from an X-ray free-electron laser (XFEL) in order to understand the kinetics of the phase transformation of $\mathrm{MgO}$.

In the second crossover region, the specific internal energy $\left(E-E_{0}\right)$ and temperature $(T)$ data along the Hugoniot were determined directly from the experiments. From the data points of shot numbers 33524 and 33684, we roughly estimated the molar specific heat, $C_{v}$ in $\mathrm{J} / \mathrm{K} \mathrm{mol}$, of the $\mathrm{MgO}$ on the basis of its definition, $C_{v}=\Delta E / \Delta T$, and we obtained an extremely high value of $\sim 101 \mathrm{~J} / \mathrm{K}$ mol [36]. This value is approximately equal to $12 R$, which is much higher than the Dulong-Petit limit, where the $R$ is the gas constant. This indicates that $\mathrm{MgO}$ undergoes a phase transformation into a system with a higher number of degrees of freedom than the solid in this region, and that significant endothermic reactions occur to cancel the shock-induced temperature increase. Melting, decomposition, and molecular dissociation are the most probable chemical reactions at such high-pressure and high-temperature conditions, as these can all act as an effective heat sink. The molten $\mathrm{MgO}$ might decompose and/or dissociate continuously and gradually with increasing shock temperature, behaving as an incompressible liquid such that the heat generated by shock competes with those endothermic chemical reactions. This is consistent with the fact that optical reflectivity of the molten $\mathrm{MgO}$ does not increase abruptly [17], suggesting that the sample is predominantly a molecular liquid in the upper crossover region. Ionization is also a probable reaction, but it occurs predominantly at higher shock pressures and temperatures.

\section{B. Comparison with previous experimental/theoretical studies}

The calculated melting temperatures of the B2 phase are consistent with those obtained by more empirical or approximate methods $[15,19]$. The calculated pressure range of the melting curve, which had been limited to pressures below $600 \mathrm{GPa}$ [19], has now been extended up to $1.5 \mathrm{TPa}$

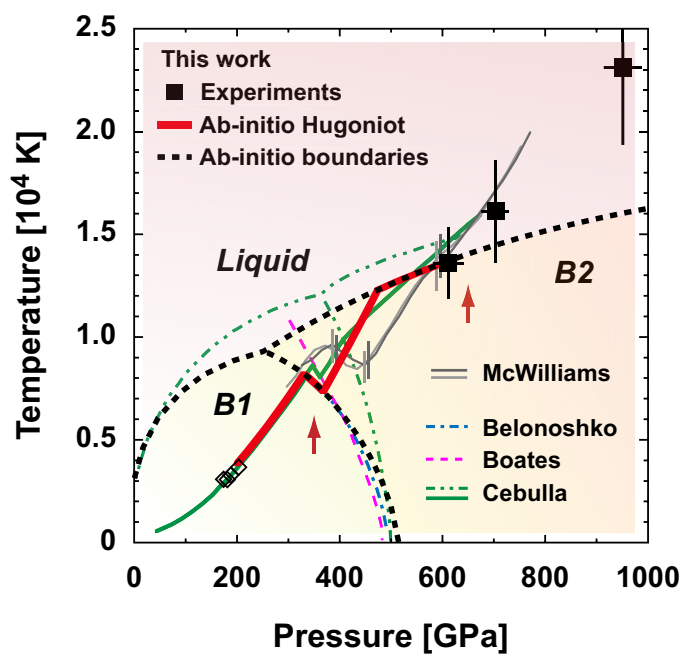

FIG. 4. (Color online) Shock pressure - temperature data of $\mathrm{MgO}$ onto the phase diagram. Present experimental results (solid squares) are shown with $a b$ initio calculation results (thick solid line), the previous Hugoniot data reported by Svendsen and Ahrens [34] (open diamonds), the decaying shock data (thin gray lines) by McWilliams et al. [17], and the ab initio Hugoniot by Cebulla and Redmer (thin solid) [20]. Phase boundaries are predicted from present $a b$ initio calculations (dotted line); Belonoshko et al. (dot-dashed) [15]; Boates and Bonev (dashed) [19]; Cebulla and Redmer (two-dot dashed) [20].

by the present study. The Hugoniot lines shown in Figs. 2-4 were calculated using the thermodynamic properties of the $\mathrm{B} 1, \mathrm{~B} 2$, and liquid phases obtained through the $a b$ initio molecular-dynamics calculations in these pressure conditions.

As shown in Figs. 2 and 3, the ab initio calculation results (thick line in the figures) are in very good agreement with the Hugoniot data. The $a b$ initio calculations predict that the B1-B2 phase transformation and the melting completion of the $\mathrm{MgO} \mathrm{B} 2$ phase occur at $\sim 350$ and $\sim 600 \mathrm{GPa}$, respectively. The two crossover regions mentioned from the experiments are consistent with the kinks corresponding to the phase boundaries of the B1-B2 and the B2-melt in the $a b$ initio Hugoniot line, respectively. The results of our $a b$ initio calculations and temperature measurements are summarized in Fig. 4, indicating that B1 phase Hugoniots are at $P<330 \mathrm{GPa}$, coexisting B1 and B2 phases are on the boundary at $330<P<370 \mathrm{GPa}$, the $\mathrm{B} 2$ phase is at $370<$ $P<470 \mathrm{GPa}$, partial melt of B2 is at $P>480 \mathrm{GPa}$, and completion of melting of B2 occurs at $P \sim 600 \mathrm{GPa}$. The two arrows in the figure represent the crossover regions shown in Figs. 2 and 3.

As reported in the recent $a b$ initio calculations for a silicate (enstatite, $\mathrm{MgSiO}_{3}$ ), it is predicted that demixing instability and phase separation into liquid $\mathrm{SiO}_{2}$ and solid $\mathrm{MgO}$ (B2-type structure) occur at 300-400 GPa and $\sim 10000 \mathrm{~K}$ [19]. The creation and fusion of such dense solid $\mathrm{MgO}$ may play a crucial role in the formation process and interior structure of extrasolar Earth-like planets, because magnesium and iron oxides will be important mantle components and may be the dominant constituent in the deep interiors of giant Earth-like planets. If the dense solid $\mathrm{MgO}$ crystallizes under different conditions to molten silicate in the magma ocean of an early 
super-Earth, it would affect the differentiation of the mantle of the planet. Mineralogical changes in the interior of a planet result in changes to the thermodynamic, mechanical, and transport properties. The behavior of warm-dense $\mathrm{MgO}$ is thus critical for understanding the dynamics of the warm dense silicate created in an exoplanet's interior and the evolution processes.

\section{SUMMARY}

In summary, we reported the principal EOS data (shock Hugoniot and temperatures) for magnesium oxide up to $\sim 1$ TPa using laser shock compression. From the behavior of $U_{s}-u_{p}$ data along the Hugoniot in the low-pressure range, we indicated that the B1 phase was stable up to $~ 350 \mathrm{GPa}$. Then, the $\mathrm{MgO} \mathrm{B} 1$ phase transforms into a warm-dense solid and liquid along the Hugoniot, revealed from the experimental data combined with $a b$ initio calculations. The experimental data were in excellent agreement with the $a b$ initio calculations.
Such warm-dense $\mathrm{MgO}$ may exist in the magma ocean of early Earth-like planets and in the deep interior of Jovian planets (e.g., the cores of Jupiter and Saturn).

\section{ACKNOWLEDGMENTS}

Laser-shock experiments were conducted under the joint research project of the Institute of Laser Engineering, Osaka University. This research was supported by grants from Grantsin-Aid for Scientific Research (Grants No. 25707041 and No. 22224012) and the Core-to-Core Program on International Alliance for Material Science in Extreme States with High Power Laser and XFEL of the Japan Society for the Promotion of Science. This work was partially supported by the Global Centers of Excellence project "Deep Earth Mineralogy" at Ehime University and the X-ray Free Electron Laser Priority Strategy Program at Osaka University from the Ministry of Education, Culture, Sports, Science and Technology. This work was also partially supported by the Genesis Research Institute, Inc. (Konpon-ken, TOYOTA).
[1] M. S. Vassiliou and T. Ahrens, Geophys. Res. Lett. 8, 729 (1981).

[2] T. S. Duffy, R. J. Hemley, and H.-k. Mao, Phys. Rev. Lett. 74, 1371 (1995).

[3] A. Zerr and R. Boehler, Nature (London) 371, 506 (1994).

[4] L. S. Dubrovinsky and S. K. Saxena, Phys. Chem. Miner. 24, 547 (1997).

[5] J. C. Jamieson, J. N. Fritz, and M. H. Manghnani, High Pressure Research in Geophysics (Center for Academic Publications Japan, Tokyo, 1982), p. 27.

[6] Y. Tange, Y. Nishihara, and T. Tsuchiya, J. Geophys. Res. 114, B03208 (2009).

[7] W. A. Bassett, T. Takahashi, H.-K. Mao, and J. S. Weaver, J. Appl. Phys. 39, 319 (1968).

[8] A. J. Cohen and R. G. Gordon, Phys. Rev. B 12, 3228 (1975).

[9] A. J. Cohen and R. G. Gordon, Phys. Rev. B 14, 4593 (1976).

[10] R. Jeanloz, T. J. Ahrens, H. K. Mao, and P. M. Bell, Science 206, 829 (1979).

[11] C. E. Sims, G. D. Barrera, N. L. Allan, and W. C. Mackrodt, Phys. Rev. B 57, 11164 (1998).

[12] N. Nishiyama, T. Katsura, K.-i. Funakoshi, A. Kubo, T. Kubo, Y. Tange, Yu-i. Sueda, and S. Yokoshi, Phys. Rev. B 68, 134109 (2003).

[13] A. typical exception of $\mathrm{B} 1$ structure materials is $\mathrm{FeO}$; R. A. Fischer, A. J. Campbell, O. T. Lord, G. A. Shofner, P. Dera, and V. B. Prakapenka, Geophys. Res. Lett. 38, L24301 (2011).

[14] D. Alfè, M. Alfredsson, J. Brodholt, M. J. Gillan, M. D. Towler, and R. J. Needs, Phys. Rev. B 72, 014114 (2005).

[15] A. B. Belonoshko, S. Arapan, R. Martonak, and A. Rosengren, Phys. Rev. B 81, 054110 (2010).

[16] N. de Koker and L. Stixrude, Geophys. J. Int. 178, 162 (2009).

[17] R. S. McWilliams, D. K. Spaulding, J. H. Eggert, P. M. Celliers, D. G. Hicks, R. F. Smith, G. W. Collins, and R. Jeanloz, Science 338, 1330 (2012).

[18] F. Coppari, R. F. Smith, J. H. Eggert, J. Wang, J. R. Rygg, A. Lazicki, J. A. Hawreliak, G. W. Collins, and T. S. Duffy, Nat. Geosci. 6, 926 (2013).

[19] B. Boates and S. A. Bonev, Phys. Rev. Lett. 110, 135504 (2013).
[20] D. Cebulla and R. Redmer, Phys. Rev. B 89, 134107 (2014).

[21] Los Alamos Shock Hugoniot Data, edited by S. P. Marsh (University of California Press, Berkeley, CA, 1979).

[22] T. Duffy and T. J. Ahrens, J. Geophys. Res. 100, 529 (1995).

[23] N. Ozaki, T. Sano, M. Ikoma, K. Shigemori, T. Kimura, K. Miyanishi, T. Vinci, F. H. Ree, H. Azechi, T. Endo, Y. Hironaka, Y. Hori, A. Iwamoto, T. Kadono, H. Nagatomo, M. Nakai, T. Norimatsu, T. Okuchi, K. Otani, T. Sakaiya, K. Shimizu, A. Shiroshita, A. Sunahara, H. Takahashi, and R. Kodama, Phys. Plasmas 16, 062702 (2009).

[24] D. G. Hicks, T. R. Boehly, P. M. Celliers, J. H. Eggert, E. Vianello, D. D. Meyerhofer, and G. W. Collins, Phys. Plasmas 12, 082702 (2005).

[25] M. D. Knudson and M. P. Desjarlais, Phys. Rev. Lett. 103, 225501 (2009).

[26] Y. B. Zel'dovich and Y. P. Raizer, Physics of Shock Waves and High-Temperature Hydrodynamic Phenomena (Academic, New York, 1966).

[27] P. M. Celliers, D. K. Bradley, G. W. Collins, D. G. Hicks, T. R. Boehly, and W. J. Armstrong, Rev. Sci. Instrum. 75, 4916 (2004).

[28] J. E. Miller, T. R. Boehly, A. Melchior, D. D. Meyerhofer, P. M. Celliers, J. H. Eggert, D. G. Hicks, C. M. Sorce, J. A. Oertel, and P. M. Emmel, Rev. Sci. Instrum. 78, 034903 (2007).

[29] D. G. Hicks, T. R. Boehly, J. H. Eggert, J. E. Miller, P. M. Celliers, and G. W. Collins, Phys. Rev. Lett. 97, 025502 (2006).

[30] P. M. Celliers, P. Loubeyre, J. H. Eggert, S. Brygoo, R. S. McWilliams, D. G. Hicks, T. R. Boehly, R. Jeanloz, and G. W. Collins, Phys. Rev. Lett. 104, 184503 (2010).

[31] D. M. Ceperley and B. J. Alder, Phys. Rev. Lett. 45, 566 (1980).

[32] T. Tsuchiya and J. Tsuchiya, Proc. Natl. Acad. Sci. (USA) 108, 1252 (2011).

[33] Y. Usui and T. Tsuchiya, J. Earth Sci. 21, 801 (2010).

[34] B. Svendsen and T. J. Ahrens, Geophys. J. Int. 91, 667 (1978).

[35] B. G. Hyde and S. Andersson, Inorganic Crystal Structures (Wiley, New York, 1989), p. 430.

[36] The $\Delta E$ between these data points is $\sim 2.55 \times 10^{5} \mathrm{~J} / \mathrm{mol}$. 